\title{
Accurate Coordinates and 2MASS Cross Identifications for (Almost) All Gliese Catalog Star
}

\author{
John Stauffer, ${ }^{1}$ Angelle M. Tanner, ${ }^{2}$ Geoffrey Bryden, ${ }^{3,4}$ Solange Ramirez, ${ }^{3}$ Bruce Berriman, ${ }^{3}$ \\ David R. Ciardi, ${ }^{3}$ Stephen R. Kane, ${ }^{3}$ Trisha Mizusawa, ${ }^{3}$ Alan Payne, ${ }^{5}$ Peter Plavchan, ${ }^{3}$ \\ Kaspar von Braun, ${ }^{3}$ PAMEla Wyatt, ${ }^{3}$ and J. DaVy KirkPatrick ${ }^{6}$ \\ Received 2010 April 30; accepted 2010 June 11; published 2010 July 22
}

\begin{abstract}
We provide precise J2000, epoch 2000 coordinates, and cross-identifications to sources in the 2MASS Point Source Catalog for nearly all stars in the Gliese, Gliese-Jahreiss, and Woolley catalogs of nearby stars. The only Gliese objects where we were not successful are two Gliese sources that are actually QSOs; two proposed companions to brighter stars, which we believe do not exist; four stars included in one of the catalogs but identified there as only optical companions; one probable plate flaw; and two stars that simply remain unrecovered. For the 4251 recovered stars, 2693 have coordinates based on Hipparcos positions, 1549 have coordinates based on 2MASS data, and 9 have positions from other astrometric sources. All positions have been calculated at epoch 2000 using proper motions from the literature, which are also given here.
\end{abstract}

Online material: color figures, enhanced table

\section{INTRODUCTION}

The Gliese-Jahreiss (1991) Catalog of Nearby Stars (also known as the CNS3) is the primary source list for those working on the properties of nearby stars. It was intended to include all stars known or thought to be located within about $25 \mathrm{pc}$ of the Sun at the time of the last update to the catalog. Because this is a very active research topic, the catalog is now significantly out of date from a variety of standpoints. Most obviously, there have been many new stars identified over the past 20 years with estimated distances closer than 25 pc (cf. Reid \& Cruz 2002; Jao et al. 2005; Henry et al. 2006). Less obviously, the positional and other data provided in the CNS3 do not include the new information provided in hundreds of papers published since 1989, requiring those who are interested in these stars often to do significant amounts of detective work in order to place each star in context. This is particularly true for the $>200$ stars in the CNS3 catalog whose listed positions were inaccurate by $>30^{\prime \prime}$ (and sometimes up to $\sim 2^{\prime}$ ), making attempts to obtain new data for these stars particularly challenging. As discussed in $\S 2$,

\footnotetext{
${ }^{1}$ Spitzer Science Center, Pasadena, CA 91125.

${ }^{2}$ Department of Physics and Astronomy, Georgia State University, Atlanta, GA 30302

${ }^{3}$ NASA Exoplanet Science Institute, California Institute of Technology, Pasadena, CA 91125.

${ }^{4}$ Jet Propulsion Laboratory, Pasadena, CA 91109.

${ }^{5}$ Australian National University, Mount Stromlo Observatory, Canberra, ACT, Australia.

${ }^{6}$ Infrared Processing and Analysis Center, California Institute of Technology, Pasadena, CA 91125.
}

there have been several versions of the nearby stars catalog. When we discuss the stars contained in these catalogs, we will use the term "Gliese stars," even though this is not entirely correct or precise.

As part of the work to create the NASA/IPAC/NExScI Star \& Exoplanet Database $^{7}$ (NStED), we have attempted to update one facet of the data in the CNS3. Specifically, we have derived accurate J2000 positions for (almost) every Gliese star, and where possible, we have cross-identified each Gliese star to the Two Micron All Sky Survey (2MASS) catalog. In $\S 2$, we provide a brief history of the Catalog of Nearby Stars. In $\S 3$, we describe our methodology for deriving J2000 coordinates for stars in the CNS3; an Appendix gives detailed information on individual stars where significant detective work was needed. Section 4 compares the new positional information with older results and presents the 2MASS photometric data as a color-color diagram with details given for outliers.

\section{HISTORY OF THE CATALOG OF NEARBY STARS}

The Gliese catalog (Gliese 1957), compiled by Wilhelm Gliese of the Astronomisches Rechen-Institut (ARI) in Heidelberg, had its beginnings in the 1950s as a list of stars within $20 \mathrm{pc}$ of the Sun. The initial catalog included 915 single and double stars, for a total of 1094 components. Star numbers in the catalog were ordered by right ascension. Resolved double stars were designated by the letters A and B (and C or higher if necessary). The second version of the catalog was published in

\footnotetext{
${ }^{7}$ At http://nsted.ipac.caltech.edu.
} 
1969 (Gliese 1969). The distance limit for this version was increased to $22.2 \mathrm{pc}$ (parallax $\left.\geq 0.045^{\prime \prime}\right)$. Because of revisions to parallax estimates that occurred over time, about 200 stars in the 1969 catalog had final parallax estimates that were in fact less than $0.045^{\prime \prime}$. There were 1529 single and multiple stars in the 1969 catalog, for a total of 1890 components. In order to maintain the original Gliese star numbers and still keep the numbering system ordered by right ascension (R.A.), new numbers were created by adding a decimal place: e.g., Gl 59.1 and 59.2 have right ascensions between those for Gl 59 and Gl 60 . The next installment to the nearby stars catalog was the Catalogue of Stars Within Twenty-Five Parsecs of the Sun (Woolley et al. 1970, Wo70), which was described by those authors as an extension of the Gliese catalog. New stars added by these authors were given the numbers from 9001 to 9850 , with the 9000 series stars also ordered by R.A. These new stars are sometimes referenced as Wo 9xxx. A number of the new stars in the Gliese (1969) and Wo70 catalogs have duplicate names (e.g., Gl 78.1 is Wo 9062; Gl 83.1 is Wo 9066). Next, Gliese \& Jahreiss (1979, hereafter referred to as GJ79) published a supplement to the nearby stars catalog. Two sets of stars were included in the supplement. Table 1 of that paper contained 294 new stars thought to have parallaxes $\geq 0.045^{\prime \prime}$; their numbers are GJ 1001 through GJ 1294 B, again ordered by R.A. Table 2 in GJ79 contained 159 stars with uncertain distance data (labeled as "Suspected Nearby Stars"); they were numbered GJ 2001 through GJ 2159. Finally, what was labeled a "Preliminary 3rd Version" of the Gliese catalog was published in 1991 (hereafter referred to as GJ91) by Gliese \& Jahreiss. The parallax limit for this catalog was $0.039^{\prime \prime}$, corresponding to $25.6 \mathrm{pc}$. This catalog contains 3803 stars, including most of the supplementary stars discussed above, and 1388 new stars. These new stars were indicated as NN 3001 through NN 4388 in the CNS3, with the expectation that these numbers would be superceded in the final version of the catalog. That did not happen, however, and these stars are now often referred to as GJ rather than NN. A few stars from previous catalogs do not appear in the CNS3 (e.g., Wo 9007), presumably because later data indicated that they did not meet the imposed parallax limit. In this article we attempt to derive accurate J2000 coordinates for all stars included in any of the above-mentioned nearby star catalogs (as opposed to just the stars in GJ91). In general, if a possible binary companion was identified in one of the nearby star catalogs but was determined to be only an optical companion, we do not provide coordinates for the companion. Also, in general, we do not include binary companions discovered since the publication of GJ91; in almost all cases, these companions have accurate positions reported in the literature.

\section{POSITIONAL DATA FOR GLIESE STARS}

The data available for stars that were added to the nearby stars catalogs varied greatly according to the exact pedigree of the star. In some cases, the positional data were of high qual- ity (accuracy to a few arcseconds). In many other cases, however, the positions were poorly known. Often, this was captured in the catalog by only listing the positions to the nearest arcminute or by not listing any proper motion. In GJ91, 455 stars have their declination (decl.) listed to 1 arcminute precision; another 48 stars have no listed proper motion. All of the other R.A. $\mathrm{s}$ in GJ91 are listed only to integer seconds of time precision. Many of the stars that have their declinations listed to 0.1 arcminute precision actually have positions known to much lower accuracy.

\subsection{Automated Recovery of Positions and Validation}

About $60 \%$ of the Gliese stars are easily cross-matched to stars in the Hipparcos catalog based either on a close coordinate match or on aliases to other catalog names provided in the Gliese catalogs and the Hipparcos Input Catalog (HIC). These stars therefore have accurate J2000 positions and proper motions as determined by Hipparcos, and with only a couple of exceptions (which we discuss in the Appendix) we have simply adopted the Hipparcos astrometry. To aid in additional identifications, we used those epoch 1991.25 positions and proper motions to predict positions at 2MASS epoch and determined the 2MASS counterparts for each Hipparcos star.

A few Gliese stars were present in the HIC, but did not yield good fits from the Hipparcos mission data and therefore do not have Hipparcos (output) catalog data. We used the data in the HIC and Gliese catalogs and Digitized Sky Survey (DSS) and 2MASS finding charts to identify these stars and determine their 2MASS cross-identifications (cross-IDs) and J2000, epoch 2000 positions. A few additional stars with very poor positional data in the Gliese or Woolley catalogs were discovered by us to be associated with Hipparcos stars during our detective work. Where warranted, we have included a discussion of these stars in the Appendix.

For the 1555 Gliese stars without Hipparcos positions, we adopted an automated process for finding J2000 positions. To start the process, we used the 1950 positions and proper motions to predict J2000, epoch 2000 positions. We then used the IRSA Gator service ${ }^{8}$ to identify all 2MASS stars within $2^{\prime \prime}$ of these positions. We then queried the Bakos et al. (2002), Salim \& Gould (2003), and Lepine et al. (2005) catalogs of high proper motion stars for position matches to the 2MASS stars. For all position matches, we compared aliases in the high proper motion catalogs to those in the Gliese catalogs. Where the aliases indicated that we had found the correct 2MASS counterpart to the Gliese star, we also made sure that the optical-near-IR colors were reasonable. For binary Gliese stars, we insured that the correct binary component was matched to the correct 2MASS source. Accurate J2000 positions for $\sim 800$ stars were derived with the automated process.

\footnotetext{
${ }^{8}$ At http://irsa.ipac.caltech.edu.
} 
The remaining approximately 700 Gliese stars were handled manually. Charts found from the first and second epoch DSS and from 2MASS were examined carefully. In most cases, the Gliese stars have high enough proper motions and distinctive enough colors that it was possible to identify the star unambiguously from these charts. The IRSA Gator service was again utilized to determine the name, J2000 (2MASS epoch) position, and $J H K_{s}$ photometry for these Gliese stars. Where an unambiguous match was not apparent, we consulted articles referencing the star (as listed by SIMBAD) for additional finding charts or other identification clues. For stars with Gliese position errors greater than $30^{\prime \prime}$ or other issues where serious detective work was required, we provide a brief synopsis of our process in the Appendix.

Figure 1 illustrates some of the difficulties posed in identifying the positions for three individual systems. The Hipparcos coordinates for GJ 563.2 AB, for example, are unreliable (panel A). In other cases, proximity of two similar brightness (but unassociated) stars has led to misidentifications in the literature (GJ 4327; panel B) or will lead to confusion in the future due to the proper motion of the Gliese star causing it to pass very near the line of sight to another star (GJ 3999 A; panel C).

Table 1 provides the $\mathrm{J} 2000$ positions and 2MASS data for the 4251 stars for which we were successful in identifying their positions. ${ }^{9}$ (Note that there are only 4106 lines of data, since some binary pairs have fully blended positional/photometric information.) Most positions are from Hipparcos (2693 stars) or from 2MASS (1549); only 9 stars have positions from other catalogs, as listed in Table 1. Table 2 provides information for the remaining 11 objects where we either could not determine a position or believe the object is nonstellar.

\subsection{Gliese Catalog Key (Table 1)}

As described in $\S 1$, the nearby star catalogs have evolved as new nearby stars were identified and original members were dropped based on new estimates of their distances that placed them outside the adopted distance limit. In Table 1 we have chosen to include nearly all stars that appear in any of the nearby star catalogs: Gl69, GJ79, GJ91, and Wo70. That is, a star that was present in the 1969 Gliese catalog but not in the 1991 Gliese-Jahreiss catalog will still be present in our Table 1. Some distant giants (>100 pc; see Table 3) and Pleiades cluster members ( 133 pc; "g" in Table 1 under Comments) remain in the table in spite of their nominally large distances. Only 11 objects previously identified as nearby stars are excluded from Table 1 : 2 sources that are actually QSOs; 2 proposed companions to brighter stars, which we believe do not exist; 4 stars that are only optical companions; 1 probable plate flaw; and 2 stars that

\footnotetext{
${ }^{9}$ Only representative lines of Table 1 are shown here; for a full listing, see http://nsted.ipac.caltech.edu/data/browse/Gliese_Positions.html.
}

simply remain unrecovered. These misidentified objects are listed in Table 2. The columns for Table 1 are described here:

Column 1: Star Name. The prefix for each star name is an indicator of the original source catalog. Gl: from the Gliese (1969) catalog; GJ: not in Gl69 but present in GJ79 or GJ91; Wo: not in any of G169, GJ79, or GJ91, but in Wo70. In some cases, binary component letters have been added to the original names, based on companion identification subsequent to GJ91. Such cases are marked by a "d" flag in the comments (column [12]).

Column 2: Alternative Name. Where a given star has two nearby star catalog names, the second name appears here. If there is no nearby star catalog name and a Hipparcos name is available, it is provided here.

Columns 3 and 4: Positions. R.A. and decl. are given for equinox and epoch 2000. For all but a few stars, the positions are based on Hipparcos or 2MASS data (as noted in column [12]). For most binary stars with separations of $5^{\prime \prime}$ or less, we list the binary pair on one row of the table with an $\mathrm{AB}$ designation. For binaries with Hipparcos positions for each component and separations $<5$ ", each star has its own row in the table, but we generally associate both components to the same (unresolved) 2MASS object.

Columns 5 and 6: Proper Motions. The proper motions in the R.A. and decl. directions in " $\mathrm{yr}^{-1}$, generally come from Tycho-2, Hipparcos, or GJ91, in that order of precedence. For the very small number of stars lacking reliable proper motions in any of these three catalogs, a handful of proper motion measurements from the USNO-B1.0 (Monet et al. 2003) and UCAC3 (Zacharias et al. 2010) catalogs have also been included. For some binaries, the proper motion of the individual components has been compromised by their proximity, particularly for Hipparcos measurements. To correct for this, we have enforced the alignment of proper motion vectors for binary components within $10^{\prime \prime}$ of each other.

Columns 7-10: 2MASS Name and Photometry. These columns list the 2MASS designation associated for each star and the corresponding $J(1.2 \mu \mathrm{m}), H(1.6 \mu \mathrm{m}), K_{s}(2.2 \mu \mathrm{m})$ 2MASS photometry. Those binary companions with separations $\lesssim 5^{\prime \prime}$ have the same photometry listed for each component. Where the 2MASS photometry for a binary star is likely to be blended, we indicate this with a "f" flag in column (11).

Column 11: Comments. Comment flags listed here are explained in the Table 1 footnotes.

Column 12: Coordinate Source. This column identifies the source of the position of the star, either the Hipparcos or 2MASS catalogs, or an additional source such as the LSPM catalog (Lepine et al. 2005).

\section{PROPERTIES OF THE COMPLETE GJ SAMPLE}

\subsection{Positional Accuracy}

We assert that we have determined arcsecond accuracy coordinates for all but a handful of the Gliese stars. Compared to 
G] 563.2AB

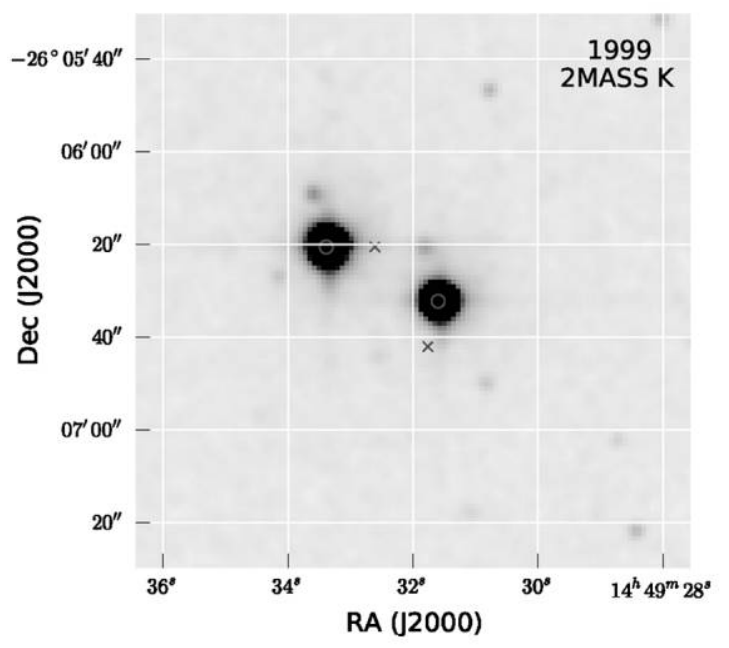

G] 4327

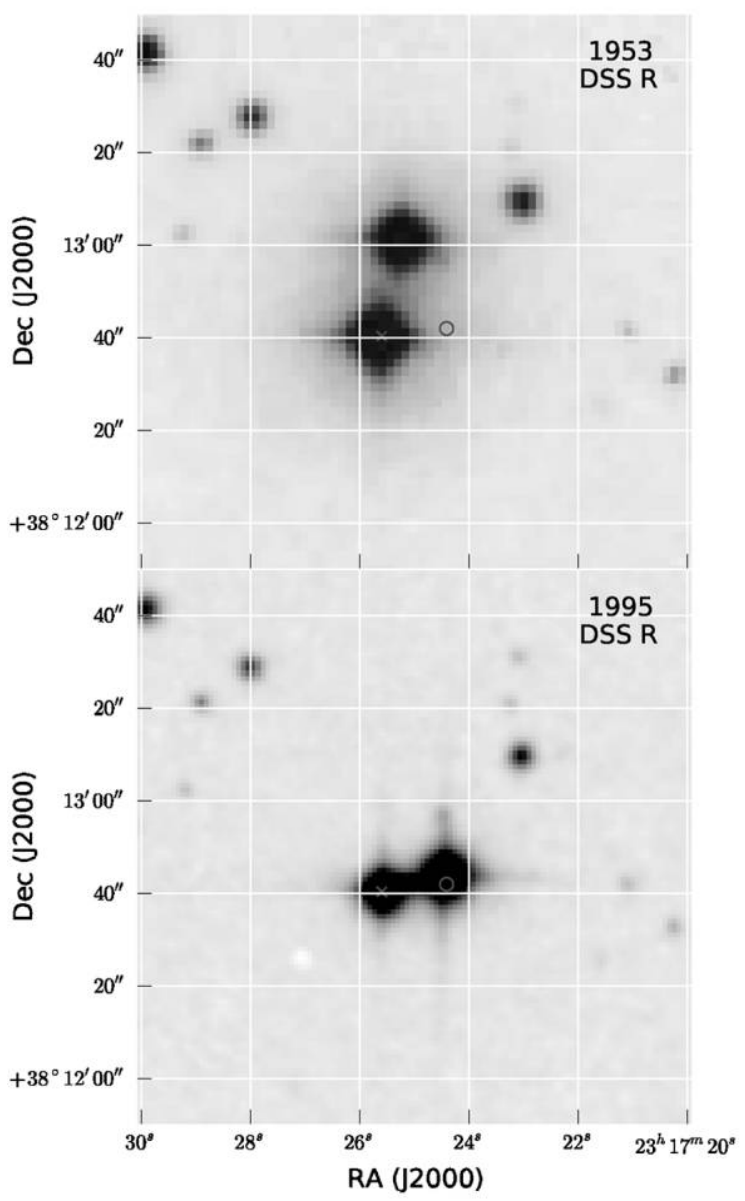

G] 3999A/4000B

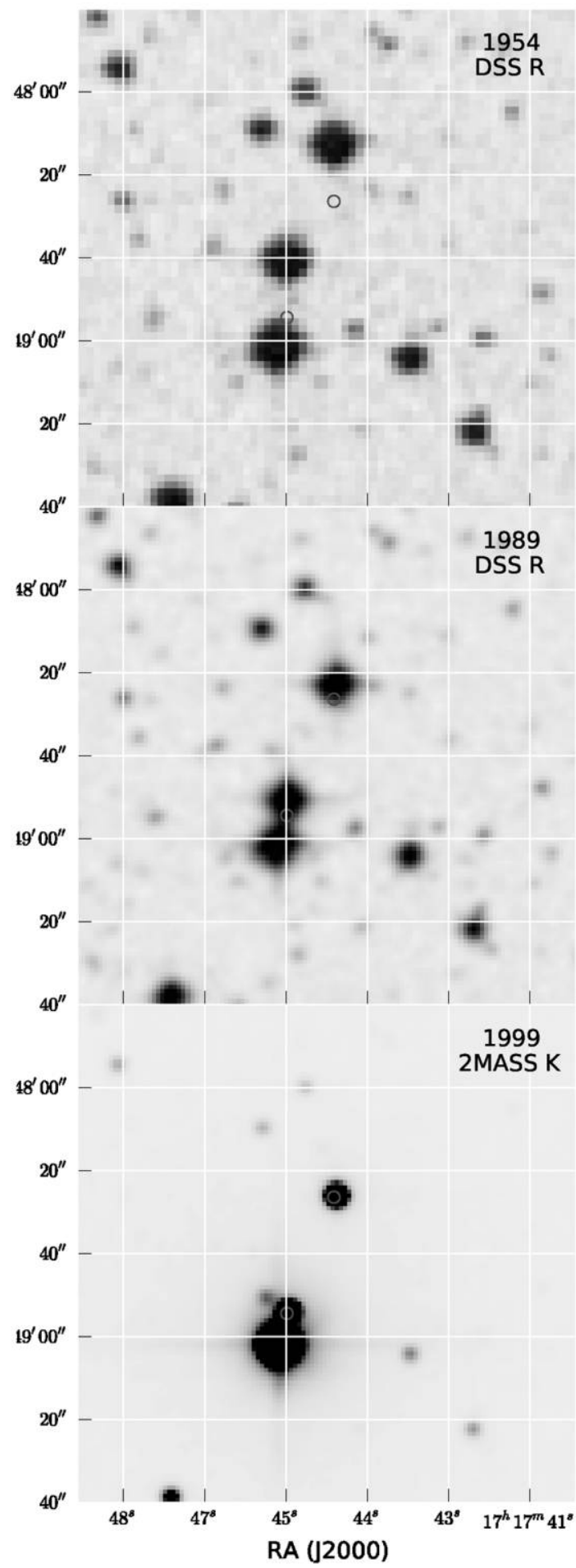

FIG. 1.-Illustrative images of three fields. In each case, a 2' by $2^{\prime}$ region is shown around the target(s) of interest. The stars' J2000 positions (from Table 1) are shown as open circles. Panel $A$ shows the 2MASS $K$ band image for GJ $563.2 \mathrm{AB}$. The corresponding Hipparcos positions (projected to epoch 2000; shown as crosses) are clearly inconsistent. In Panel B, the 1953 and 1995 DSS images show GJ 4327 approaching a nonmoving field star HIP 114994 (indicated by a cross), causing confusion in their identification. Panel C shows GJ 3999 A/4000 B at three epochs-1954, 1989, and 1999. As the pair moves southward, GJ 3999 A will soon become nearly coincident with the nonmoving field star. See the Appendix for additional details on each of these systems. See the electronic edition of the PASP for a color version of this figure. 


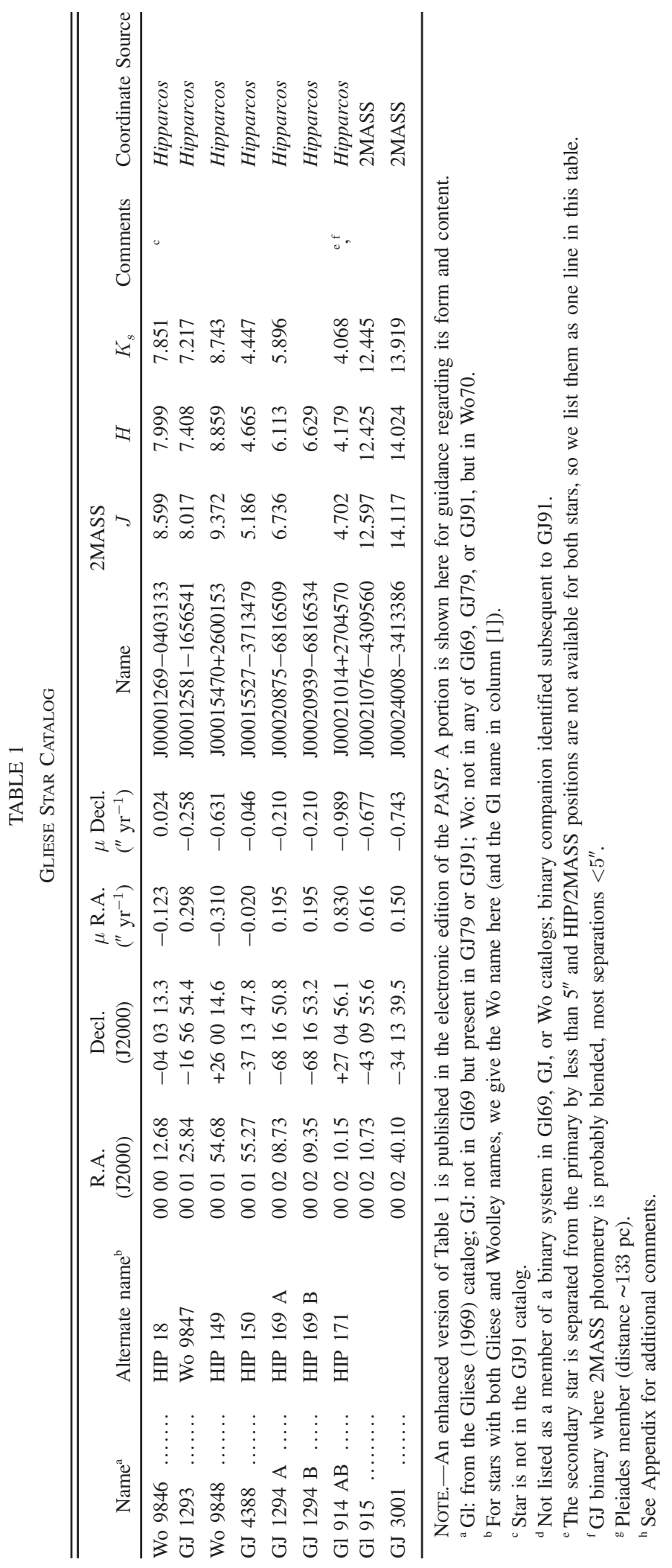


TABLE 2

GLIESE "STARs" NOT IN TABLE 1

\begin{tabular}{|c|c|c|c|c|}
\hline Name & Alternate name & $\begin{array}{l}\text { R.A. } \\
(\mathrm{J} 2000)\end{array}$ & $\begin{array}{l}\text { Decl. } \\
(\mathrm{J} 2000)\end{array}$ & Reason not in Table 1 \\
\hline Gl 127.1 B & & & & Companion does not exist ${ }^{\mathrm{a}}$ \\
\hline Gl 452 B & LHS 2470a & & & Only an optical companion \\
\hline $\begin{array}{l}\text { Gl } 678.1 \text { B } \ldots \ldots \\
\text { Gl } 732 \mathrm{~B} \quad \ldots \ldots\end{array}$ & Wo 9592 B & 173021.96 & +053306.5 & $\begin{array}{l}\text { Only an optical companion } \\
\text { Companion does not exist }{ }^{\mathrm{a}}\end{array}$ \\
\hline Gl $863.1 \mathrm{C} \ldots \ldots$ & Wo $9788 \mathrm{C}$ & & & Only an optical companion \\
\hline GJ $1154 \mathrm{~B}$ & & 121418.17 & +00 3729.7 & Only an optical companion \\
\hline GJ $2087 \ldots \ldots$ & LHS 6219 & & & Probable plate flaw ${ }^{\mathrm{a}}$ \\
\hline GJ 3063 & & & & Unable to recover this star ${ }^{\mathrm{a}}$ \\
\hline GJ 3750 & QSO B1246+586 & 124818.78 & +58 2028.7 & BL Lac object $\mathrm{t}^{\mathrm{b}}$ \\
\hline GJ 3848 & 7C $1424+2401$ & 142700.39 & +234800.0 & BL Lac object ${ }^{\mathrm{b}}$ \\
\hline GJ 4103 & & & & Unable to recover this star \\
\hline
\end{tabular}

${ }^{a}$ See Appendix for details.

${ }^{\text {b }}$ Fleming et al. 1993

the original source catalogs, SIMBAD provides improved positions for many Gliese stars and, indeed, $78 \%$ of our positions agree with SIMBAD (as of 2010 April) to within an arcsecond. Nevertheless, there are still many Gliese stars whose positional listing in SIMBAD is significantly in error. As a symptom of the less well-known positions, SIMBAD only lists 2MASS crossids for 3293 of the 4251 Gliese stars, whereas we report 2MASS ids and photometry for all but 14 . The number of stars whose positions in Table 1 differ from SIMBAD by $>10^{\prime \prime}$ is 315 , with 52 of these different by more than $1^{\prime \prime}$. An additional 85 stars from Table 1 are either not listed in SIMBAD or do not have coordinates there. For the Gliese stars listed in SIMBAD, Figure 2 shows the difference between our coordinates and those provided by SIMBAD.

\subsection{MASS Photometry}

For the 2MASS photometry presented in Table 1, Figure 3 compares the $H-K_{s}$ and $J-H$ colors. Most stars follow a well-defined color sequence extending from white dwarfs in the

TABLE 3

OutLIERS IN THE 2MASS Color-Color Distribution (Fig. 3)

\begin{tabular}{|c|c|c|c|c|c|c|}
\hline Name & $J-H$ & $H-K_{s}$ & $J$ & $H$ & $K_{s}$ & Comment \\
\hline Gl $166 \mathrm{~B}$ & -0.137 & 0.125 & 9.849 & 9.986 & 9.861 & White dwarf; $8^{\prime \prime}$ away from $\mathrm{C}$, which is 3 mag brighter \\
\hline Gl $168.1 \ldots \ldots \ldots$ & 0.784 & 0.239 & 11.833 & 11.049 & 10.810 & \\
\hline Gl 425 B $\ldots \ldots$. & 0.779 & 0.033 & 6.638 & 5.859 & 5.826 & $4^{\prime \prime}$ binary \\
\hline Gl $552.1 \quad \ldots \ldots \ldots$ & 1.171 & 0.153 & 8.597 & 7.426 & 7.273 & 2MASS $J$ is bad; see Leggett 1992 \\
\hline Gl 618 B $\ldots \ldots$. & 0.304 & 0.270 & 8.339 & 8.035 & 7.765 & $8 "$ binary; B much fainter than A \\
\hline Gl 752 B $\ldots \ldots$. & 0.682 & 0.461 & 9.908 & 9.226 & 8.765 & VB10; very late-type M dwarf \\
\hline GJ $1230 \mathrm{~B} \quad \ldots$. & 0.829 & 0.257 & 8.860 & 8.031 & 7.774 & $5^{\prime \prime} \mathrm{dM}-\mathrm{dM}$ binary \\
\hline GJ $2110 \ldots \ldots$. & 0.815 & 0.273 & 7.434 & 6.619 & 6.346 & Distant giant $(>100 \mathrm{pc})$ \\
\hline GJ 2129 & 0.913 & 0.258 & 8.131 & 7.218 & 6.960 & Distant giant $(>100 \mathrm{pc})$ \\
\hline GJ 3107 & 0.856 & 0.273 & 8.434 & 7.578 & 7.305 & Probably a giant \\
\hline GJ $3140 \quad \ldots \ldots$. & 0.903 & 0.313 & 8.287 & 7.384 & 7.071 & Distant giant $(>100 \mathrm{pc})$ \\
\hline GJ $3231 \mathrm{~B} \quad \ldots$. & 0.639 & -0.035 & 8.029 & 7.390 & 7.425 & 2MASS $K_{s}$ flagged as nonoptimal; $20^{\prime \prime}$ binary \\
\hline GJ $3276 \quad \ldots \ldots \ldots$ & 0.990 & 0.491 & 12.757 & 11.767 & 11.276 & LH 190 (probably Hyad); faint late-type M dwarf \\
\hline GJ 3364 & 0.347 & 0.393 & 11.659 & 11.312 & 10.919 & \\
\hline GJ $3361 \mathrm{~B} \quad \ldots$. & 0.533 & -0.077 & 6.661 & 6.128 & 6.205 & $5^{\prime \prime}$ binary \\
\hline GJ $3517 \quad \ldots \ldots \ldots$ & 0.743 & 0.527 & 11.212 & 10.469 & 9.942 & LHS 2065; very late-type M dwarf \\
\hline GJ 3655 & 0.695 & 0.498 & 11.928 & 11.233 & 10.735 & LHS 2397A; very late-type M dwarf \\
\hline GJ 3849 & 0.765 & 0.481 & 11.990 & 11.225 & 10.744 & LHS 2924; very late-type M dwarf \\
\hline GJ $4370 \quad \ldots \ldots$ & 0.141 & 0.512 & 8.841 & 8.700 & 8.188 & 2MASS $J \& K_{s}$ flagged as nonoptimal \\
\hline Wo 9383 A ..... & 1.076 & 0.219 & 9.148 & 8.072 & 7.853 & $3.4^{\prime \prime}$ binary \\
\hline Wo 9383 B ..... & 1.236 & 0.205 & 9.366 & 8.130 & 7.925 & $3.4^{\prime \prime}$ binary \\
\hline Wo $9584 \mathrm{C} \ldots .$. & 0.356 & 0.235 & 9.020 & 8.664 & 8.429 & 2MASS $K_{s}$ flagged as nonoptimal \\
\hline
\end{tabular}




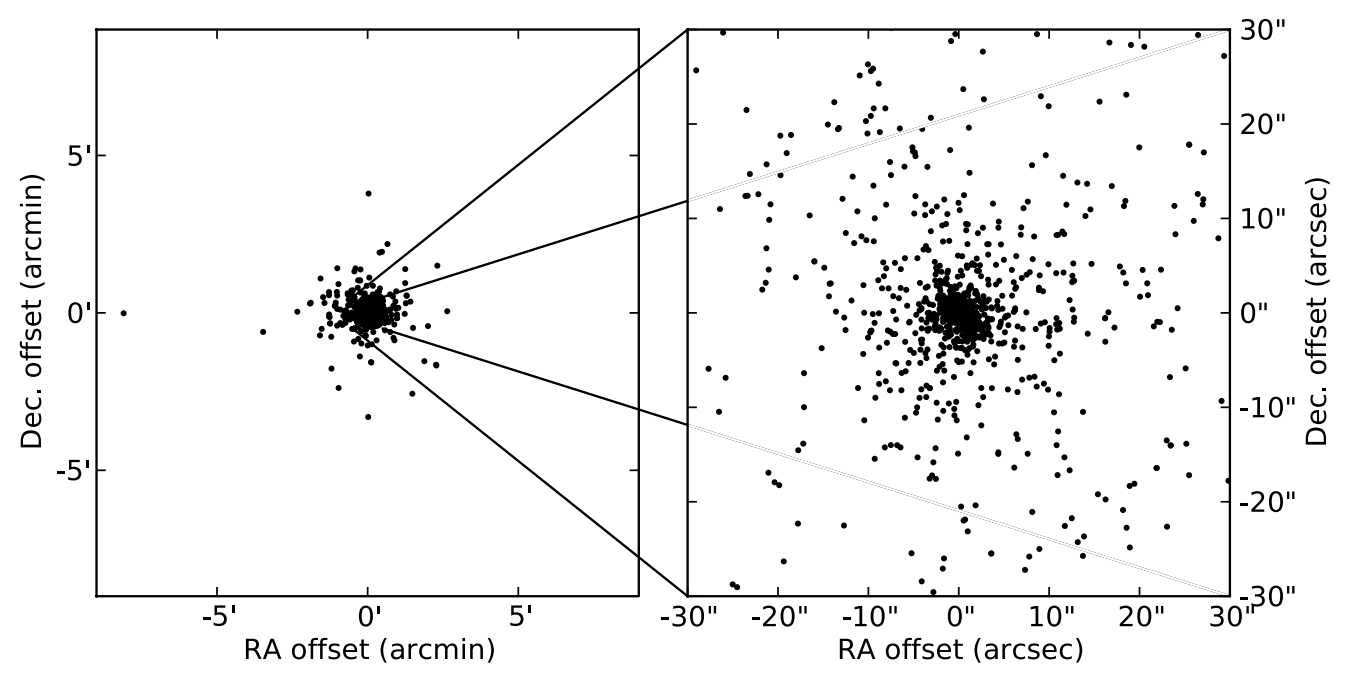

FIG. 2.-For Gliese stars listed in SIMBAD (as of 2010 April), we show the differences between our calculated positions and those given by SIMBAD. The left panel displays stars with offsets of several arcminutes, while the right panel zooms in to show differences on a finer scale. The majority of the stars fall in the center of the plots, with positions agreeing to better than $1^{\prime \prime}$.

lower left up into the main sequence which follows an L-shaped central locus of point. The densest pack of stars corresponds to main-sequence $\mathrm{M}$ stars, with the very latest-type stars trailing to the upper right. Outliers (Fig. 3) are detailed in Table 3.

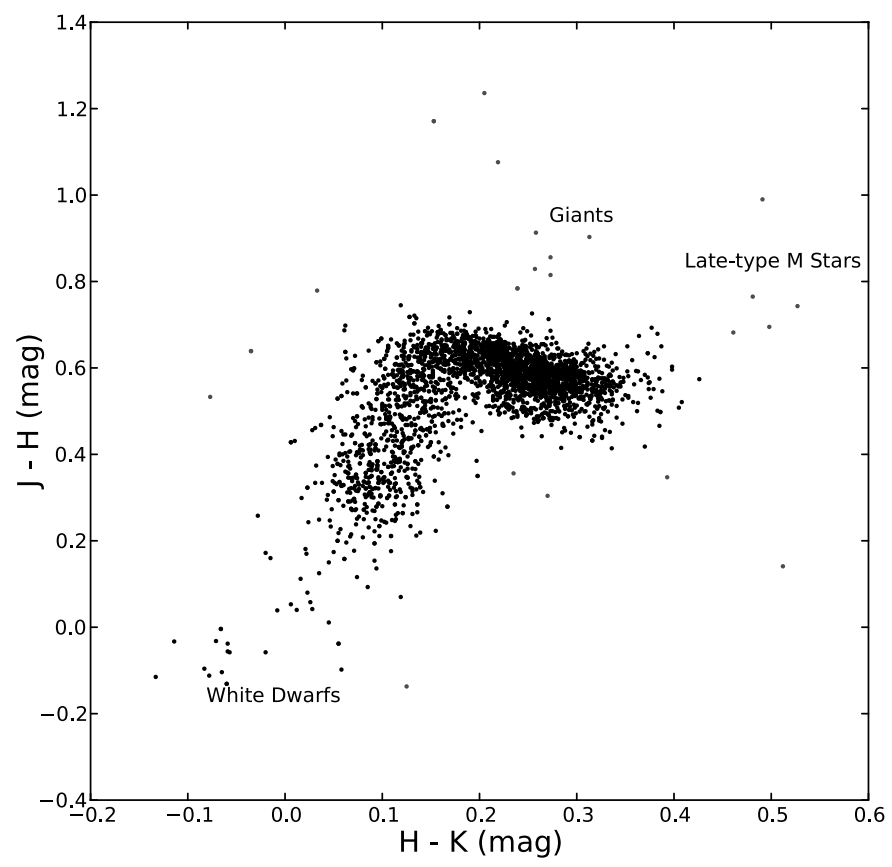

FIG. 3.-Near-IR color-color diagram. For the 2MASS photometry presented in Table $1, J-H$ is plotted vs. $H-K_{s}$. Most stars follow a well-defined color sequence. Outliers from the main distribution (points) are detailed in Table 3. See the electronic edition of the PASP for a color version of this figure.

\section{CONCLUSIONS}

Because they are nearby and hence relatively bright, Gliese catalog stars often serve as template objects. In particular, nearby stars are among the most important targets in the sky for surveys of planets and planetary debris (e.g., Butler et al. 2006; Bryden et al. 2006). Their attractiveness as survey targets comes not only from their relative brightness but also because they subtend the greatest angles for high-resolution imagingof their disks and, potentially, planets. Not surprisingly, many of the most exciting planetary discoveries have been for Gliese stars, including the first detected debris disk (Vega/GJ 721;Aumann 1984), the first resolved disk ( $\beta$ Pic/GJ 219; Smith \& Terrile 1984), the first planet detected around a main-sequence star (51 Peg/GJ 882; Mayor et al. 1995), the firstmultiple-planet system ( $v$ And/GJ 61; Butler et al. 1999), and the first imaged planet (Fomalhaut/ GJ 881 ; Kalas et al. 2008). Future surveys for planets via astrometric wobble (Unwin et al. 2008), infrared radial-velocity variation (Bean et al. 2010), or direct imaging (Beichman et al. 2007) will similarly focus on our closest neighbors. It is therefore important that the properties of these stars be tabulated in an accurate and homogeneous fashion.

While the quality of astronomical observations is normally limited foremost by the brightness of the targets, the proximity of the stars in the Gliese catalogs results in unique limitations; their high proper motion and large number of well-separated binaries create serious difficulties in proper identification of the stellar positions. The net result is a surprisingly high degree of inaccuracy in the positions of stars that would otherwise be expected to be the among the best in the sky. We have taken a first step toward providing better data for the Gliese stars by 
providing accurate $\mathrm{J} 2000$ positions and $J H K_{s}$ photometry for nearly all Gliese catalog stars. In Paper II of this series, we will provide published $B V R I$ photometry for a large fraction of these stars.

We would like to acknowledge a careful reading of the manuscript by an anonymous referee. This publication makes use of data products from the NASA/IPAC/NExScI Star \& Exoplanet
Database (NStED), the Two-Micron All Sky Survey (2MASS), the NASA/IPAC Infrared Science Archive (IRSA), and the SIMBAD and VizieR databases operated at CDS, Strasbourg. Some of the research described in this publication was carried out at the Jet Propulsion Laboratory, California Institute of Technology, under a contract with the National Aeronautics and Space Administration.

\section{APPENDIX}

Gl 63-Examination of DSS and 2MASS images shows two stars near the GJ91 position at the epoch of the 2MASS images. GJ 63 is the westernmost, high proper motion component of this optical binary, and is 2MASS J01382162+5713571.

G1 127.1 B-Listed as an AB pair in the GJ91 catalog, with a separation of 8", but no position angle indicated. GJ 127.1 A is a well-known star with a Hipparcos position, so there is no difficulty identifying A. The Gliese catalog lists $V=11.43, B-V=0.01$ for A, $V=14.73, B-V=0.62$ for B. Caballero \& Solano (2007), however, state that "The status of the hypothetical companion GJ 127.1 B is unknown." We examined all available online images of the field (including 2MASS, where the A-B contrast should be best) but found no sign of a $\mathrm{B}$ component, at any position angle, with a separation of 8 " from A. We are therefore skeptical that $\mathrm{B}$ exists.

Gl 130-Examination of DSS and 2MASS finding charts shows a star of the right magnitude, color, and proper motion within a few arcseconds of the position indicated in the GJ91 catalog. This star is 2MASS J03122972-3805204. We believe this is the correct ID. This disagrees with the position listed in SIMBAD (by about 30"), which is derived from Bakos (2002). Our choice agrees with the position reported by Rousseau \& Perie (1997).

Gl 293-Examination of the 2MASS and DSS finding charts shows a star of the right proper motion at $\sim 30^{\prime \prime}$ from the position indicated in the GJ91 catalog. This star is 2MASS J075308146747314.

Gl $421 \mathrm{AB}-\mathrm{Gl} 421 \mathrm{~A}$ and B form a visual binary separated on the sky by about $20^{\prime \prime}$ (mostly east-west); Gl $421 \mathrm{C}$ is $\sim 4$ mag fainter and $\sim 1.5^{\prime}$ northwest from AB. The Hipparcos J2000, epoch 2000 coordinates for the AB pair are offset by nearly 5 " from the 2MASS epoch 2000 coordinates. Using the average of the positions for G1 $421 \mathrm{C}$ in UCAC3, HST-GSC2.3.2, and Lick NPM (all agree to within $0.2^{\prime \prime}$ ) and measuring the offset from C to A on DSS and 2MASS plates, we derive an epoch 2000 position for A of 1115 19.42, -18 08 40.2, which is nearly identical to the 2MASS epoch 2000 position. We therefore adopt the 2MASS positions for Gl $421 \mathrm{~A}$ and B. We also adopt our measured proper motions for A and B $\left(0.14^{\prime \prime}\right.$ yr in R.A., $0.72^{\prime \prime} \mathrm{yr}$ in decl.), which agree well with the proper motion for C, but disagree with the Hipparcos proper motion in R.A. (though this does not explain the position offset between Hipparcos and 2MASS).

Gl 451 B-Discovered by van de Kamp in 1968 as an apparent flare star companion, separated by $2^{\prime \prime}$ from A. Not detected since 1968 to our knowledge (e.g., Heintz 1984).

Gl 452 B-Identified as a faint, blue companion about 8 " to east of $\mathrm{Gl} 452$ A by Luyten. We see a star of the right magnitude and location in the 1954 blue DSS plate. However, it is not common proper motion with Gl 452 A. Reid \& Gizis (2005) report they could not detect B at the expected position with the Keck I guide TV (at epoch 2002), with an estimated limiting magnitude of $R \sim 21$. We suspect Gl 452 B does not exist, at least as a physical companion to A.

Gl 534.1 B-Location of B relative to A given incorrectly in GJ91. Correct relative position given in the Catalog of Components of Double and Multiple Stars (CCDM). Identification of B and confirmation of common proper motion with A made by examination of DSS and 2MASS finding charts.

Gl 549 C - Gl 549 was listed as a triple system in Gl69, with the $\mathrm{C}$ component being located almost $1.5^{\circ}$ north of the $\mathrm{AB}$ pair. In GJ91, the $\mathrm{C}$ component was no longer listed as a member of the system. $\mathrm{C}$ is real, and does have a proper motion quite similar to the $\mathrm{AB}$ pair. However, its parallax is different from that of the AB pair (Gliese \& Jahreiss 1988), and its spectral type and apparent magnitude are also inconsistent with forming a physical system with $\mathrm{AB}$.

Gl 563.2 AB-Alternate names for this binary are HIP 72509 and HIP 72511, and Luyten Half-Second Catalogue (LHS) 379 and LHS 380. Normally we would have just accepted the Hipparcos positions. However, the J2000, epoch 2000 positions derived from the Hipparcos data are clearly inconsistent with what is seen in the DSS and 2MASS charts (see Fig. 1). The Hipparcos positions indicate that the two stars are separated by $11^{\prime \prime}$ in R.A. and $21.5^{\prime \prime}$ in decl. However, visual examination of the finding charts clearly indicates the stars have separations more like $20^{\prime \prime}-25^{\prime \prime}$ in R.A. and of order $10^{\prime \prime}$ in decl. We have chosen to adopt the 2MASS positions for the system, and note that these positions agree with what is given in the Hubble Space Telescope (HST) GSC 2.2 and by the Carlsberg Meridian Catalog, while they disagree by $10^{\prime \prime}-15^{\prime \prime}$ with the Hipparcos positions. 
Gl $601 \mathrm{~B}$ - The notes to G69 identify this star with L 153157 , with a position offset from the A component of 157" at position angle (P.A.) $258^{\circ}$. A star with the expected motion is visible near the predicted position. The only astrometric catalog we can find for this star is the UCAC3, which lists 1554 43.49, -632614.8 (epoch 1998.3).

Gl $629.2 \mathrm{~B}-$ The notes section of G69 indicates that this is a common proper motion companion to Gl 629.2 A. Giclas et al. (1960) provide 1950 coordinates and a finding chart for Gl $629.2 \mathrm{~B}$ under the designation G17-27. That finding chart allows easy identification of this star as 2MASS J163529020357571.

Gl 732 B-This star probably does not exist. Separation from A listed as about 10" and about 3.3 mag fainter. Proper motion given as about $1.0^{\prime \prime} \mathrm{yr}^{-1}$ to P.A. $=161^{\circ}$. Examination of the DSS and 2MASS charts shows no evidence for a companion to A. We could find no postdiscovery evidence in the literature that $\mathrm{B}$ exists.

G1 $734 \mathrm{AB}$ - The GJ91 catalog indicates that B is separated by $5.2^{\prime \prime}$ at P.A. $=21^{\circ}$ from $\mathrm{A}$, with $\mathrm{B}$ being about 2.5 mag fainter than A in the visual. Similar information is listed in the Washington Double Star Catalog (WDS) and CCDM. Visual inspection of the DSS and 2MASS images allows easy identification of A, but no strong evidence for the presence of B. The 1952 DSS images show a hint of extension in the north-south direction, with a hint of a second set of diffraction spikes northward of the main spikes, as expected. The 2MASS PSC shows two sources when queried, the brighter being $\mathrm{A}$ and the fainter displaced from A by about 4" to the north-northeast. This second source is barely detected by 2MASS, but is at the approximate position expected for B. We adopt the 2MASS coordinates; a better measurement would be useful.

G1 762-Nothing present within 30" of the GJ91 coordinate. Examination of the DSS and 2MASS charts shows a star of the right (relatively large) proper motion and colors about $45^{\prime \prime}$ from the cataloged position. No other star of similar magnitude is within $2.5^{\prime}$.

G1 774 AB-The Hipparcos input catalog lists GJ 774 A as an alias to HIC 98811 A, providing a J2000 coordinate of 2004 $02.1,-653558.1$. However, that is approximately the correct coordinate for GJ $774 \mathrm{~B}$ (and about 20" from its true position). HIP 98811 A should instead be aliased to GJ 774 B. The GJ91 coordinate and proper motion allow the $\mathrm{J} 2000$ position to be estimated to better than $30^{\prime \prime}$ accuracy, and the proper motion is large enough to be distinctive, thus allowing easy identification of the correct star.

Gl 781.1 AB-The GJ91 catalog provides individual R.A. and decl. values for both components, with the secondary indicated as being 3 seconds west and $18^{\prime \prime}$ north of the primary. When the GJ91 1950 coordinates are precessed, the proper motion corrected to J2000, epoch 2000 , and those positions checked versus the 2MASS images, there are two relatively bright stars within a few arcseconds of where they are predicted to be: $\mathrm{A}$, at $200748.0,-314530.0$ (2000); and B, at 2007 45.0, - 3145 14. (2000). Comparing the 2MASS and DSS images shows those stars to both have the proper motions expected $\left(0.8^{\prime \prime} \mathrm{yr}^{-1}\right.$ toward P.A. $\left.159^{\circ}\right)$. The only problem is that $\mathrm{Gl}$ 781.1 A was included in the Hipparcos input catalog as HIC 99150. A star was found by Hipparcos at almost exactly the predicted HIC location, and subsequently HIP 99150 was given the Hipparcos 1997 position of $200744.98,-314514.4$ (2000). Unfortunately, based on the data in GJ91, this star is not Gl 781.1 A but is instead Gl 781.1 B. SIMBAD adopted the Hipparcos identification, and called the star at 2007 48.0, - $314530 \mathrm{Gl} 781.1 \mathrm{~B}$. We prefer to retain the positional reference data from the GJ catalog itself, making the A component the southeastern star of the binary. The two stars have very similar luminosities.

Gl 806.1 B-The A component of this system is a $V=2.45$, $\mathrm{K}$ giant. According to GJ91, the B component is located 6 seconds west and 6" south from A, and is listed as a $V=13.4$, M4 dwarf. We could not confirm B by its proper motion using the DSS and 2MASS charts because the image of A is so bright. However, the 2MASS images show two stars at about the expected position for B. Neither star is in the online 2MASS PSC. However, both stars are in the 2MASS "working database." One of this pair is too blue to be an M star. The brighter and more northwest of this pair does have near-IR colors compatible with being an M4 dwarf. That star is at $204606.39+335806.1$ (2000), observed by 2MASS on 1999 May 16 , with $J=9.456$, $H=8.889, K=8.649$ (uncertainties of $0.031,0.015$, and 0.009 ; data from the 2MASS working database).

Gl 871.1 AB - This binary appears in the G169 catalog, but not in GJ91. Spectral types are listed as M3 and M4. The positions in Gl69 were only given to a precision of 0.1 minute of time, and $1^{\prime}$ of declination; the pair has negligible proper motion. No star appears within $1^{\prime}$ of the stated position on the DSS and 2MASS charts, but the binary is clearly identifiable on those charts (based on relative position and colors) about 2 ' west of the cataloged position.

GJ $1001 \mathrm{AB}$-GJ 1001 was listed as a single star in the GJ79 update to the Gliese catalog. Using the coordinates from GJ79, no compatible object is within $1^{\prime}$ on the DSS or 2MASS charts. However, a star with the right proper motion $\left(1.6^{\prime \prime} \mathrm{yr}^{-1}\right.$ toward P.A. $\left.=155^{\circ}\right)$ is visible about $2^{\prime}$ south of the expected position. A common proper motion companion is also visible in those charts to the west of the primary. This pair is now referenced as GJ $1001 \mathrm{~A}$ and $1001 \mathrm{~B}$ (see Goldman et al. 1999). Both stars have 2MASS counterparts, which we have adopted for our position reference.

GJ 1022-Examination of DSS and 2MASS charts showed no star within 1' of the expected position from GJ91. However, a star matching the cataloged proper motion $\left(1.119^{\prime \prime} \mathrm{yr}^{-1}\right.$ to P.A. $\left.=94^{\circ}\right)$ is easily identifiable on those charts about $1.5^{\prime}$ from the GJ91 position. The 2MASS colors of that star (2MASS 
J00492903-6102326) are compatible with the late-M spectral type given for this star by GJ91.

GJ 1167 B-GJ 1167 A is easily identified in DSS and 2MASS charts, within $15^{\prime \prime}$ of the position given by GJ91. GJ91 states that "comp B doesn't exist." However, in the GJ79, a GJ $1167 \mathrm{~B}$ is listed (at a position about 80" east and $3^{\prime}$ north of the A position). A star is present in the DSS and 2MASS charts within about $15^{\prime \prime}$ of the GJ79 prediction, and with proper motion between the 1955 epoch DSS and 2000 epoch 2MASS charts essentially identical (to the eye) to the A component. The photometry for B is significantly bluer than for A, however, which seems unphysical since A is $2-4$ mag brighter than B. This suggests the pair is not a physical binary, in agreement with the statement in GJ91.

GJ 1287 -Despite a true position more than $30^{\prime \prime}$ from what is given in GJ79, this star is easily identified on DSS and 2MASS charts due to its large proper motion $\left(0.9^{\prime \prime} \mathrm{yr}^{-1}\right.$ to P.A. $\left.=98^{\circ}\right)$. It was not included in GJ91 because newer distance estimates place it beyond $25 \mathrm{pc}$.

GJ 2013-Examination of DSS charts shows no good candidate within 1' of the GJ79 position. We identified the star using the finding chart in Eggen (1968). This star is not included in GJ91 because the revised distance estimate places it beyond 25 pc.

GJ 2014-An accurate position for this star is provided by Costa et al. (2006). The corresponding 2MASS object is 2MASS J00495863-2624055. The near-IR and $V-K$ colors agree with that expected for the $B-V$ color listed in GJ79. This star was not included in GJ91.

GJ 2023-This is a very faint star with quite small proper motion. It is therefore not possible to identify it unambiguously using the DSS and 2MASS charts. Friedrich et al. (2000) were able to identify it and obtained spectra confirming it as a white dwarf. Using the coordinates provided in that article, we identify GJ 2023 as 2MASS J01295612-3055098.

GJ 2052-This is a faint, bluish star with little proper motion. Only one star is present within $30^{\prime \prime}$ of the predicted position on the DSS charts. That star is not present in the 2MASS PSC, because it is too faint. A blue star with nearly the same $V$ magnitude as listed in GJ79 and located within 5" of the GJ79 position is present in the Lick Northern Proper Motion Survey (Klemola et al. 1987). We adopt their position.

GJ 2087-There is no star in the DSS or 2MASS charts at the J2000 GJ91 position and Bakos et al. (2002) claim this is a plate flaw.

GJ 2133 - This star is located in a very crowded field. No object with the expected proper motion and color is present on the DSS and 2MASS charts within 1' of the cataloged position. A star with the expected characteristics is present on those charts about $1^{\prime}$ west and $30^{\prime \prime}$ south of the cataloged position. That star is 2MASS J17540302-3440177.

GJ $2154 \mathrm{AB}$-Examination of the DSS charts showed no stars matching the expected properties within $1^{\prime}$ of the cataloged position. However, the binary is easily identifiable about $1.5^{\prime}$ away, based on the relatively large proper motion and the appropriate separation and position angle.

GJ 3063 - This should be a relatively bright $(V=11.9) \mathrm{M} 3$ star of low proper motion according to the GJ91 catalog. However, only two stars appear in the 2MASS images near the cataloged position, and neither are particularly red. No star of approximately the right properties is present in, for example, the USNO B1 or HST GSC within several arcminutes of the expected position. The only alias in GJ91 is to Sm177 (Smethells 1974), and SIMBAD provides no literature references. We were unable to recover this star.

GJ 3085-No star of the expected properties is located on the DSS charts within $1^{\prime}$ of the position listed in GJ91. A finding chart is provided in Pesch \& Sanduleak (1978); based on that chart, we identify GJ 3085 as being located about $2.2^{\prime}$ north and $1^{\prime}$ east of the cataloged position. Its near-IR colors are consistent with the "m" spectral type provided in GJ91.

GJ 3096-No star of the expected properties is located on the DSS charts within $1^{\prime}$ of the position listed in GJ91. A finding chart is provided in Pesch \& Sanduleak (1978); based on that chart, we identify GJ 3096 as being located about 1.2' north and $0.4^{\prime}$ west of the cataloged position. Its near-IR colors are consistent with the "m" spectral type provided in GJ91.

GJ 3101-No star of the expected properties is located on the DSS charts within $1^{\prime}$ of the position listed in GJ91. Reid et al. (1995) list a position for GJ 3101 that is more than $5^{\prime}$ different from that in GJ91. Comparison of the DSS chart at the Reid et al. position to a finding chart in Pesch \& Sanduleak (1978) allows identification of GJ 3101 (aka PS244). This star is 2MASS J01360872-2652161; the near-IR colors are consistent with the "m" spectral type listed in GJ91.

GJ 3397-Inspection of the DSS images shows a star with the correct colors and proper motions about $0.8^{\prime}$ south and $0.1^{\prime}$ east of the coordinates in the GJ91 catalog. This star is 2MASS J06322189-6957445.

GJ 3521-Examination of the DSS charts shows no appropriate star at the cataloged position. A star with the expected proper motion, magnitude, and colors is present about $1^{\prime}$ west and $10^{\prime \prime}$ south of the GJ91 position. This star is 2MASS J08550473-7135480.

GJ 3592-We used the finding chart provided by Eggen (1969) to identify this star on the 2MASS charts. This object is 2MASS J10145842-5611114. This position agrees with that given by Wegner (1973).

GJ 3618-The very large proper motion of this star $\left(1.5^{\prime \prime} \mathrm{yr}^{-1}\right)$ makes its identification on DSS and 2MASS charts easy despite the more than $1^{\prime}$ error in the cataloged position.

GJ 3999 A/4000 B-This is a binary separated by $30^{\prime \prime}$ at P.A. $=338^{\circ}$. Identification is made difficult because GJ 3999 A is passing very near a much brighter field star at 2MASS epoch (see Fig. 1). Its distinctive proper motion and colors make the identification secure. 
GJ 4012-No star evident on the DSS and 2MASS charts within $2^{\prime}$ of the cataloged position. We used a finding chart from Luyten (1949), under the alias L270-37, to identify the star on the DSS charts, and to confirm its proper motion by comparison to 2MASS charts. Our 2MASS-based J2000 position is within $\sim 5^{\prime \prime}$ of the position listed by Downes et al. (2001).

GJ 4028-Cataloged position in error by $\sim 1.5^{\prime}$. High proper motion makes identification on DSS and 2MASS charts secure.

GJ 4078-There is a star 2.2' east and 1' south of the GJ91 coordinate in the DSS images, whose colors and proper motion match those expected for GJ 4078. This star is 2MASS J18495119-5726486. SIMBAD lists the star at this position as LHS 3413, one of the aliases given for GJ 4078 in the GJ91 catalog.

GJ 4088-In the GJ91 catalog, GJ 4088 is aliased to LP 57180. LP 571-80 is aliased to HIC 93047 in the HIC. However, the position given in the HIC is considerably in error, and presumably as a result no useful data were obtained by Hipparcos for this star. The coordinates given in the GJ91 catalog are in error by less than $30^{\prime \prime}$, allowing easy identification of the correct star based on its proper motion and magnitude.

GJ 4103-Very little information for this star is given in GJ91. An alias is given to LP 336-6 (ver. 1 of the New Luyten Two-Tenths Catalogue [NLTT]), and the listed position and proper motion are taken directly from the NLTT (or from the Bruce Proper Motion Survey [BPM] catalog, where it is listed as BPM94000). However, no object with the listed proper motion is present within $2.5^{\prime}$ of the listed position. A star of somewhat similar position and proper motion is present several arcminutes north of the listed position, but that star is identified in the NLTT as LP 336-7, and we do not believe there is enough information to equate that star to GJ 4103. We do note that there is a third NLTT star somewhat nearby in space whose listed red and blue magnitudes and proper motion size and direction are exactly equal to those for LP 336-6; this other object is LP 3362 . A barely resolved binary of the appropriate general brightness and proper motion is present on the DSS and 2MASS charts at about the position listed for LP 336-2; this pair is 2MASS $\mathrm{J} 19050788+3237547$ and 2MASS J19050756+3237526. Weis (1987) also noted the exact duplication of information in the NLTT for these two LP stars, and that no obvious candidate was present at the listed position for LP 336-6. Our conclusion (similar to Weis), is that either LP 366-6 does not exist, or it is probably the same object as LP 336-2.

GJ 4112-The DSS images show a bright star 30" east and 8" north of the GJ91 coordinates whose proper motion and colors match those expected for GJ 4112. This star is 2MASS J19340394-5225144. Pokorny et al. (2004) list the star at this position as having a proper motion consistent with that given in the GJ91 catalog, and indicate this star is L275-26, one of the aliases listed in the GJ91 catalog for GJ 4112.

GJ 4133-High proper motion star $\left(1.08^{\prime \prime} \mathrm{yr}^{-1}\right)$. The DSS images have a bright star $1.1^{\prime}$ east and $45^{\prime \prime}$ south of the GJ coor- dinates whose proper motion and colors match those expected for GJ 4133. This star is 2MASS J20053482-1056545. SIMBAD matches this star to LHS 483 and LP 754-16, both aliases for GJ 4133 given in the GJ91 catalog.

GJ $4187 \mathrm{~A} / 4188 \mathrm{~B}$-True position slightly more than 30" different from GJ91 coordinates, but binary easily identifiable on DSS and 2MASS charts.

GJ 4189 A/4190 B-These two binary components have a separation of $4^{\prime \prime}$ in the GJ91 catalog and a P.A. of $341^{\circ}$ in the WDS catalog. The stars are of a similar spectral type and are resolved in the DSS images where it is clear they share a common proper motion. Using the information in the WDS catalog we conclude that GJ 4190 B is the more northerly star. This disagrees with the GJ91 information (which traces back to the NLTT), but agrees with the double star measures of Worley (1962).

GJ 4191-The GJ91 catalog indicates this star is a white dwarf with a relatively high proper motion. Examination of DSS images shows a blue star 90" east and 15" north of the GJ position that has about the right proper motion. That star is 2MASS J21193652-5550144.

GJ 4193-DSS and 2MASS charts show no appropriate match within $1^{\prime}$ of the input coordinate. There is a star of the right magnitude and color about $20^{\prime \prime}$ east and 1.6' south of the GJ coordinate. This star is 2MASS J21261422-4227320. Hawley et al. (1996) give this as the coordinate for BPM 43997, which is one of the aliases listed for GJ 4193 by the GJ91 catalog.

GJ $4224 \mathrm{AB}-\mathrm{No}$ star is visible in the DSS or 2MASS charts within 1' of the expected position based on GJ91 1950 position and proper motion. A star with the right magnitude, colors, and proper motion $\left(0.34^{\prime \prime} \mathrm{yr}^{-1}\right.$ to P.A. $\left.=121^{\circ}\right)$ is present about $1^{\prime}$ east and 1.5' south of the GJ91 position. The GJ91 catalog does not provide P.A. or separation, but reports a difference in magnitude of 0 . On the DSS charts, the star we have identified as GJ 4224 has faintly visible double diffraction spikes, indicating a binary separated approximately north-south by a few arcseconds. Weistrop (1980) states that GJ 4224 (under the alias Sm 89) "appears double at the telescope," also indicating the separation is probably on the order of $2^{\prime \prime}-3$ ". Based on its J2000 coordinate, the star we have identified as GJ 4224 is also HIP 107711. We use the Hipparcos position for both components.

GJ 4237 A/4238 B-Listed in the GJ91 catalog as being separated by $3.4^{\prime \prime}$, but no P.A. given. The 2MASS catalog lists two stars of about the right magnitude and separation very near the GJ91 input position. These two stars are 2MASS J21553860+ 3302138 and 2MASS J21553870+3302106. The WDS indicates that B is south of A, therefore we match GJ 4237 A to 2MASS J21553860+3302138, and GJ 4238 B to 2MASS J21553870+ 3302106.

GJ 4249 - There is no star of appropriate magnitude within $2^{\prime}$ of the GJ91 position. A bright star with the appropriate (large) proper motion is, however, easily found on the DSS and 2MASS 
charts about $3^{\prime}$ east of the cataloged position. Based on the J2000 position and magnitude, this star is HIP 108890 . We adopt the Hipparcos position for GJ 4249.

GJ 4327-There are two stars with essentially equal brightness at the position indicated by GJ91. The westernmost of the pair is redder and has high proper motion to the south, as expected; we identify this star as GJ 4327. It is 2MASS J23172441+3812419. GJ91 lists LHS 3293 as an alias. Unfortunately, the HIC aliases LHS 3293 to HIP 114994, the easternmost (approximately stationary) star of the pair. We disagree with this latter alias, and instead alias LHS 3293 to the 2MASS star indicated above.

GJ 4386-There is no appropriate star within 30" of the GJ91 coordinates; however, a star of the right magnitude, color, and proper motion (based on examination of DSS and 2MASS finding charts) is present abut $60^{\prime \prime}$ south and $15^{\prime \prime}$ west of the original coordinate. This star is 2MASS J23594483-4404599. SIMBAD cross-identifies this star with LTT 9828 and LP 988-175, both aliases for GJ 4386 listed in GJ91. The $J-K$ color for this star seems much too blue for the M7 spectral type listed in GJ91. The actual spectral type is probably much earlier; Reid et al. (1995) give the spectral type as M2.

Wo 9354-The Wo70 catalog indicates that this star is Vyss 606 (Vyssotsky 1956). There is no star that matches the expected properties within $1^{\prime}$ of the input position. Only one star of about the right magnitude and color is present within about $2.5^{\prime}$ of the Woolley coordinate; it is 2MASS J11163293
+2627415. Examination of the HIC indicates that this star is HIP 55077; the HIC provides a cross-identification to Vyss 606 , confirming the identification.

Wo 9358-No star of the appropriate properties falls within $1^{\prime}$ of the Woolley catalog position. A star of the correct (but relatively small) proper motion and with the correct approximate magnitude lies about 3 ' south of the Woolley position. This star is also HIP 55605.

Wo $9540 \mathrm{C}$-The A component of this system is $V=4.2$. According to GJ91, the C component is separated from A by 7.6" at P.A. $=53^{\circ}$. C is not present in the 2MASS PSC. Because $\mathrm{A}$ and $\mathrm{B}$ are so bright, $\mathrm{C}$ cannot be identified on the DSS or 2MASS charts. However, a star in the right position relative to A is present in the HST GSC2.3.2, and we adopt the coordinate from that source for Wo $9540 \mathrm{C}$.

Wo 9785-This star is the exciting source for the planetary nebula NGC 7293. Use of IRSA's FINDER allows relatively secure identification of the star and a match to 2MASS J22293854-2050136.

Wo 9805-No star of the appropriate properties falls within $2^{\prime}$ of the Woolley catalog position. However, the Woolley catalog indicates that Wo 9805 is also Vyss 856, and the HIC crossmatches Vyss 856 to HIP 113782. We confirm that the colors and (small) proper motion of the star at the Hipparcos position agree with expectations for Wo 9805 given its spectral type of M0.5 and BVRI photometry provided in Laing (1989).

\section{REFERENCES}

Aumann, H. H. 1984, ApJ, 278, L23

Bakos, G. Á., Sahu, K. C., \& Németh, P. 2002, ApJS, 141, 187

Bean, J. L., et al. 2010, ApJ, 713, 410

Beichman, C. A., et al. 2007, in Protostars and Planets V, ed. B. Reipurth, D. Jewitt, \& K. Keil (Tucson: Univ. Arizona Press), 915 Bryden, G., et al. 2006, ApJ, 636, 1098

Butler, R. P., et al. 1999, ApJ, 526, 916

- 2006, ApJ, 646, 505

Caballero, J. A., \& Solano, E. 2007, ApJ, 665, L151

Costa, E., Méndez, R. A., Jao, W.-C., Henry, T. J , Subasavage, J. P., \& Ianna, P. A. 2006, AJ, 132, 1234

Downes, R. A., Webbink, R. F., Shara, M. M., Ritter, H., Kolb, U., \& Duerbeck, H. W 2001, PASP, 113, 764

Eggen, O. J. 1968, ApJ, 153, 723 1969, ApJ, 157, 287

Fleming, T. A., Green, R. F., Jannuzi, B. T., Liebert, J., Smith, P. S., \& Fink, H. 1993, AJ, 106, 1729

Friedrich, S., Koester, D., Christlieb, N., Reimers, D., \& Wisotzki, L. 2000, A\&A, 363, 1040

Giclas, H. L., Slaughter, C., \& Burnham, R. 1960, Lowell Obs. Bull., 4,136

Gliese, W. 1957, Astron. Rechen-Inst. Heidelberg, Ser. A, No. 8 1969, Astron. Rechen-Inst. Heidelberg, 22, 1 (G169)

Gliese, W., \& Jahreiss, H. 1991, Astron. Rechen-Inst. Heidelberg (GJ91)

1988, Ap\&SS, 142, 49
Gliese, W., \& Jahreiss, H. 1979, A\&AS, 38, 423 (GJ79)

Goldman, B. 1999, A\&A, 351, L5

Gould, A., \& Chaname, J. 2004, ApJS, 150, 455

Hawley, S. L., Gizis, J. E., \& Reid, I. N. 1996, AJ, 112, 2799

Heintz, W. D. 1984, PASP, 96, 557

Henry, T. J., et al. 2006, AJ, 132, 2360

Ivanov, G. A. 2008, Kinemat. Phys. Celest. Bodies, 24, 334

Jao, W. C., et al. 2005, AJ, 129, 1954

Kalas, P., et al. 2008, Science, 322, 1345

Klemola, A. R., Jones, B. F., \& Hanson, R. B. 1987, AJ, 94, 501

Laing, J. D. 1989, South African Astron. Obs. Circ., 13, 29

Laskar, B. M., et al. 2008, AJ, 136, 735

Leggett, S. K. 1992, ApJS, 82, 351

Lépine, S. 2005, AJ, 130, 1680

Luyten, W. J. 1949, ApJ, 109, 528

Mayor, M., \& Queloz, D. 1995, Nature, 378, 355

Monet, D. G., et al. 2003, AJ, 125, 984

Pesch, P., \& Sanduleak, N. 1978, AJ, 83, 1090

Pokorny, R. S., Jones, H. R. A., Hambly, N. C., \& Pinfield, D. J. 2004, A\&A, 421, 763

Reid, I. N., \& Cruz, K. L. 2002, AJ, 123, 2806

Reid, I. N., \& Gizis, J. E. 2005, PASP, 117, 676

Reid, I. N., Hawley, S. L., \& Gizis, J. E. 1995, AJ, 110, 1838

Rousseau, J. M., \& Perie, J. P. 1997, A\&AS, 124, 437

Salim, S., \& Gould, A. 2003, ApJ, 582, 1011

Smethells, W. G. 1974, Ph.D. thesis, Case Western Reserve Univ. 
Smith, B. A., \& Terrile, R. J. 1984, Science, 226, 1421

Unwin, S. C., et al. 2008, PASP, 120, 38

Vyssotsky, A. N. 1956, AJ, 61, 201

Wegner, G. 1973, MNRAS, 163, 381

Weis, E. W. 1987, AJ, 93, 451
Weistrop, D. 1980, AJ, 85, 738

Woolley, R., Epps, E., Penston, M., \& Pocock, S. 1970, R. Obs. Ann., 5 (Wo70)

Worley, C. E. 1962, AJ, 67, 403

Zacharias, N., et al. 2010, AJ, 139, 2184 